\title{
Survey on Wireless Sensor Network
}

\author{
Anish Bala \\ Research scholar (ECE) \\ Maharaja Ranjit \\ Singh Punjab \\ technical university \\ Punjab (152004) India
}

\author{
Amit Grover \\ Assistant Professor (ECE) \\ Maharaja Ranjit Singh \\ Punjab technical university \\ Punjab (152004)
}

\begin{abstract}
Wireless sensor network (WSN) has come out as one of the most auspicious technologies for the future. The WSN is most important application has environmental monitoring and target tracking. WSN has been enabled by advancement in technology and availability of inexpensive, small, and smart sensors lead to cost effective and easily portable WSNs. However, researchers must address numerous challenges to facilitate the worldwide deployment of WSN technology in real-world fields. This paper delineates the concept of sensor networks that has been made feasible by the convergence of micro electro-mechanical systems technology, digital electronics and wireless communication. First, the architecture of senor node is described, and a review of factors influencing the design of sensor node is provided. Then, the framework of wireless sensor network is outlined. The advancement of wireless sensor network was originally discovered for military applications like battlefield surveillance. Now a day's wireless sensor networks are used in various civilian application areas, including environment and healthcare applications, habitat monitoring, home automation and traffic control.
\end{abstract}

\section{Keywords}

WSN, Sensor

\section{INTRODUCTION}

A Wireless Sensor Network (WSN) is a special kind of wireless network constituting of small and spatial distributed autonomous devices which together sense physical phenomena them [5].

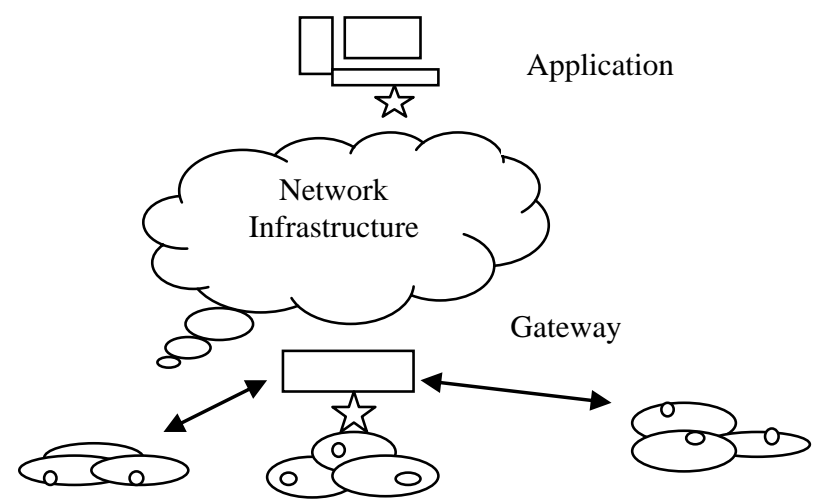

Sensor Network

Fig1: Wireless Sensor Network

It also develops the collected data adequately route them to local sink or gateway node. it consist of a numerous densely deployed sensor nodes [1].each node in the sensor network may constitute of one or more sensors, portable power supply, a low power radio and possible localization hardware, like a
GPS(Global Position System) unit or a ranging device. These nodes organize wireless transceiver so that communication and network are enabled. Additionally, the network possesses self organizing capability leally, individual nodes should be battery powered with a long lifetime and should cost very little. a key feature of such network is that their nodes and untended. Therefore, they have limited and non-replenish able energy resources. Hence, energy efficiency is an initial design discussion for these networks.

In case one or more sensor, each node in wireless sensor network is typically supplied with a radio transceiver or other communication device, and a energy source or small scale microcontroller, usually a battery. They size a single sensor node can very form Shoebox-sized node down to device the size corn of dust. Cost of sensor nodes same as variable, ranging from hundreds of dollars in a minor cents. Confide in the size of the sensor network and the convolution mandatory of individual sensor nodes.

\subsection{Type of Sensors}

There are divided into a three categories.

1.1.1Passive, Omni Directional sensor: The passive sensors sense the data without actually shape the environment by active probing. They are self powered i.e. they needed energy only to amplify their analog signal. There is no concept "direction" involved in this frequency.

1.1.2Passive, narrow-beam sensor: The sensor is passive but they have well defined concept of direction of frequency. For example 'camera'.

1.1.3Active sensor: Group of sensor heavily probe the environment, example, solar or radar sensor, they generate shock wave by small blast.

\section{ARCHITECTURE OF SENSOR NODES}

The one of most critical feature is the hardware, namely the node detonation. The node is a resource strained device capable to radio communication, sensing and narrow data processing. It's also capable of influence the environment. Power processing is low, energy as well as memory. Sensor node is commonly relaxed of four component: a power unit, one or more sensing unit and processing unit or actuating units and a transceiver.. These figures differ from the families of microcontroller, and with different vendors.

One or more power batteries, providing a $3 \mathrm{~V}-4.5 \mathrm{~V}$, with a capacity range between $1700 \mathrm{mAh}-2700 \mathrm{mAh}$. They nodes can be equipped with various sensor for acoustic, pressure, photo, temperature etc based application. All nodes may also optionally equip with an interface for plugging -in an activator for performing any mechanical actions on a function specific basis. This figure 1.2 shows the format a sensor node. 


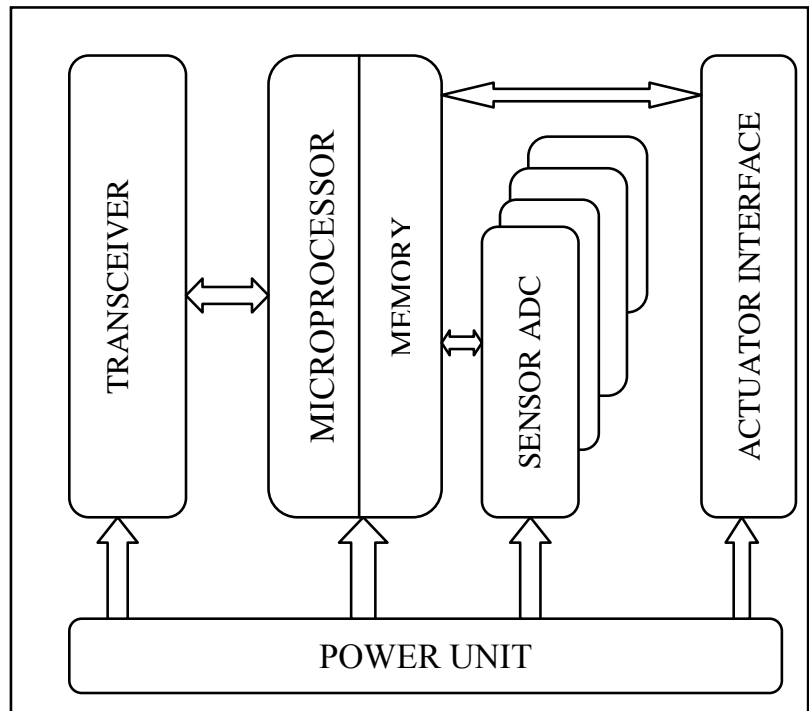

\section{FACTORS OF SENSOR NODES}

Sensor nodes main factors are microcontroller, external memory, power source or transceiver [1].

\subsection{Microcontroller}

Microcontroller performs the function, development data and controller the functionality of other feature in the sensor nodes. Alternative that can be used as a controller are: Digital signal processes, General purpose desktop microcontroller, Application specific integrated circuit and Field Programmable Gate Array Microcontroller are suitable choice for sensor node. All of the four options have their own merit and demerit. Microcontrollers are the perfect option for embedded system. Considering the flexibility to connect to other device, power consumption is less, and those devices can go to sleep state and part of controller can be active. In case microcontroller the power consumption is higher than microcontroller then it is not a correct choice for sensor node. Digital Signal Processors are applicable for broadband wireless communication. However Wireless Sensor Network, the Wireless communication should be quiet i.e., simpler, then easier to process modulation and signal processing responsibility of certain sensing of data is less complicated.

\subsection{External Memory}

An energy perspective, the relevant kind of memory are onchip of memory in a microcontroller and FLASH memory off-chip RAM is hardly used. Flash memory are used to cost and storage capacity. Memory requirement are dependent on application. They two element of memory is based on the purpose of storage

a. User memory is used for storage application relating to the personal data.

b. Program memory is commonly used for programming device.

\subsection{Power source}

Power application in the sensor node for the sensing the data. Higher energy is mandatory for data communication in sensor node [3,4]. Energy expenditure is very less for sensing and the data processing. They transmit energy of cost is $1 \mathrm{~Kb}$ for distance is $100 \mathrm{~m}$ almost the same as that the executing 3 million instruction by 100 million per second/W processor. Power is stored either used for the batteries or capacitors. Batteries the main source of power supply for sensor nodes.
Then two standard of batteries used are chargeable and nonchargeable. They are also classified give to the electrochemical material are used for electrode like NiZn(nickel-zinc), Nimh(nickel metal hydride), NiCd(nickel-cadmium), and lithium-Ion. Current sensor are advanced which are able to extreme energy from solar, vibration energy or themogernator. Considering two power saving polices used for dynamic voltage scaling (DVS) and dynamic power management (DPM). DPM take care shutting down element of sensor node which are not directly used or active. DVS scheme divided into the power levels depends on the non-deterministic workload. Varying the voltage along the frequency, and it is possible to obtain the rectangular power consumption.

\subsection{Transceiver}

Sensor is used for ISM (Industrial science medical) band that give free radio, huge spectrum allocation and global availability. The various option of a wireless transmission media are radio frequency optical communication infrared. Laser required for less energy, but they line-of-sight for communication also sensitive to atmospheric conditions. Infrared laser, no needs of antenna but is less in its broadcasting capacity. Radio frequency based on the communication is the most important that fit to the WSN applications. WSN's used for the communication frequencies between $433 \mathrm{MHz}$ and $2.4 \mathrm{GHz}$. The functionality of transmitter and receiver are combined to the single device that transceiver are used in the sensor nodes the transceiver is unique identifier. Operational states are receiver, transmitter, sleep and Idle. Current radio generation have a built-in state machines that operation perform automatically. Radio transceiver is used in four different modes: receive, transmit, sleep and idle. Almost equal to power consumed in receive mode, and radio operating in Idle mode results in power consumption. It is better to completely shut down the radio quite than in the idle mode when is not transmitting or receive. Also significant amount of power is expanding then switching from sleep mode to transmit mode to transmit a packet.

\section{FRAMEWORK OF WIRELESS SENSOR NETWORK}

The framework of wireless sensor network in the form of logical point of view, they node can be contacted through the services of the intermediate layer. They can't perform individual tasks. They shared intermediate coordinates the service within the network. it is logical to operate in the network layer but physically stand in the nodes. Each layer together conjunction with the structure constructs the sensor network application. The middleware structure is also called SINA (Sensor Information Networking architecture). SINA architecture proposes Tasking language and sensor Query as the programming interface between SINA middleware and sensor application. Operating system acts as interface between the sensor network application and hardware. Tiny OS is the fundamental based on operating system that is design for sensor network $[1,2,5]$.

Wireless sensor network constituting of a huge number of large sensor nodes, in which communicate wirelessly. The figure 1.4 shows the architecture of sensor node. These factor influence increase of the sensor node.

- Increase the complication on microchip.

- Wireless networking technologies is high performance. 
- A combination of sensor data acquisition and digital signal processing.

- Forward the evolution of micromechanical systems.

- High performance development tools.

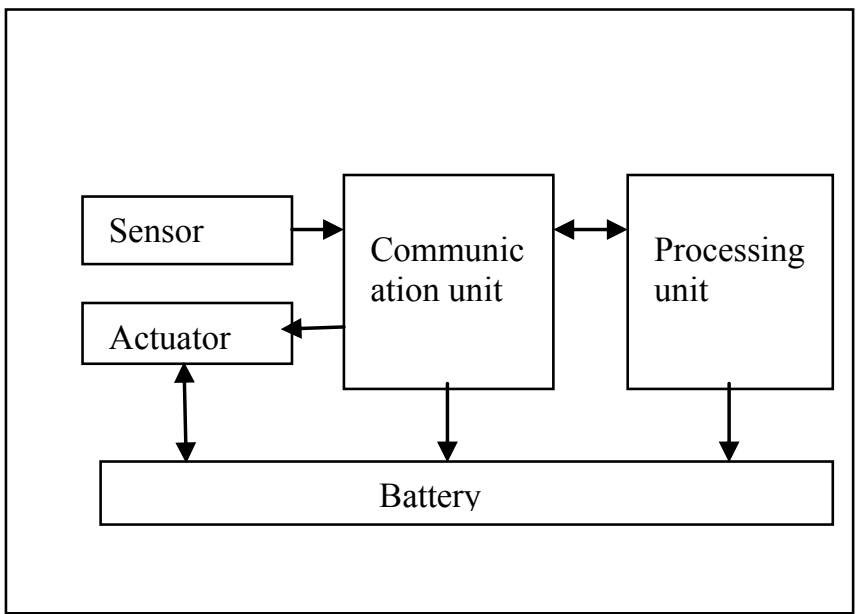

Fig 1: frameworks of sensor nodes

The sensor node can be imaginary or narrow computer, which are basic terms of their interference and component. They are consist of a processing unit with limited computing power and narrow memory, a communication device (usually radio transceiver or alternatively optical), sensor (including specific conditioning circuitry), and a power source commonly used in form of battery. base station are well known port of the WSN with higher computational, communication and energy resources.

\section{ARCHITECTURE OF WIRELESS SENSOR NETWORK}

The figure 1.5 in wireless sensor network structure constituting of one sink node and numbers of sensor nodes expand over a small geographic area. Data are transmitted in the form of sensor nodes to the sink over a multi-hop communication paradigm [7].

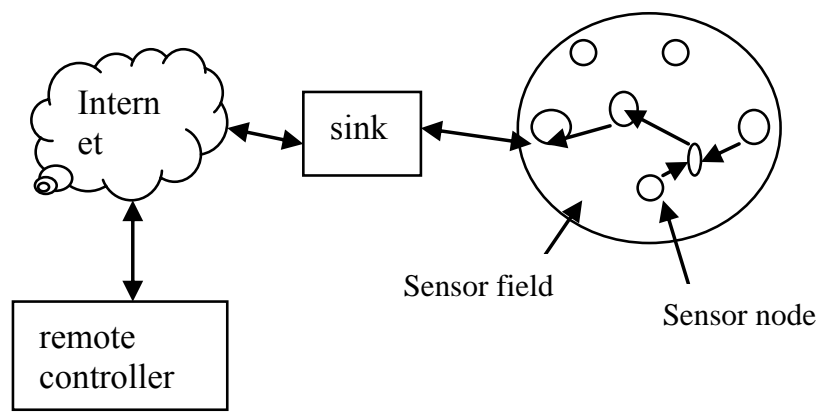

Energy expenditure for transmission of data is compared to the data processing [4]. The cost of energy is transmitted a one bit of information is almost same as that needed for processing a thousand application in a common sensor node. They energy expenditure of the sensing system are depends on the individual sensor groups. Almost, energy -saving mode focus on two subsystem: the sensing subsystem (i.e., techniques are used to lower amount of frequency and energy-expensive samples) and network subsystem energy management is arrested into the account in the operation of all single nodes. In lifetime of a sensor network can be continues jointly applying various techniques. Example, The energy efficient protocols are calculated at decreases the energy consumption during network movement. Generally, a huge amount of energy is consumed by the node factor (radio, CPU, etc.) even if they are Idle. Power management schemes are used for switching off node factor that are not temporarily needed. It focuses mainly on the network subsystem by considering duty cycle. Moreover, the main technique are applicable to reduce the energy consumption of sensor although energy cost for data acquisition i.e. sampling) cannot be neglected. Finally, announce mobility as a new energy management paradigm with the purpose of continues the network lifetime.

\section{COMPONENT OF WSN}

\subsection{Mobility of node}

Nodes of mobility create a dynamic network topology. They will be dynamically formed when two nodes are come into the communication range of each other and torn sown when they move out of range $[1,5]$.

\subsection{Abandoned operation}

In case, Sensor network have no human interference. The nodes themselves are important for the reshape in case of any changes [6].

\subsection{Dynamic network topology}

It is the important fact of the sensor network. The sensor network may be represented in three phase with respect to the maintenance. During the distributed phase, the nodes are discarded into the positions in ad hoc manner. They nodes needs to self dependent into the communication network. The post-distributed phase topology maintenance constituting induced due to the loss of the nodes, or arrival of some mobile obstacles and failure of radio links. The re-construct phase connection the deployment nodes to change the failed nodes. All of the three phases, the sensor network should able to seamlessly coordinated itself to stream data to the base station [7].

\subsection{Narrow power}

The sensor networks are highly sensitive to energy usage [3,4]. They may possible to distribute in inhospitable or hostile environments, it not be available to refresh energy sources. Hence, the energy -aware protocols/ application are desirable and energy utilization is a major issue. Energy utilization is detected at three stages, sensing, node communication and signal processing. It improve the three processes will lead to a reduction in the energy utilization.

\subsection{Large scale of deployment}

Start communication within large network, constituting of hundreds or thousands of nodes dump far apart, is not possible [6]. Further, many parameters like as noise, dispersion, interference, available bandwidth, imbalance of links and continuously changing signal strength, may complete connectively impossible even in tiny networks.

\section{QULITY OF WIRELESS SENSOR NETWORK}

There are follow different quality of the wireless sensor network.

- Large scale of formations.

- Abandoned operation

- Scale0down sensor nodes.

- Finite powers they can result are store. 
- Sharp environment conditions.

- Flexibility of nodes.

- Network topology is effective.

\section{MERIT OF WIRELESS SENSOR NETWORK}

They are following the list of merit of wireless sensor network $[2,5]$.

- In Advance low power and low cost wireless communication, microprocessor hardware, and micro sensor, further progress in ad hoc network protocols and routing, array processing and distributed signal, pervasive computing, and embedded system acquire to all sensor networking a topic of active interest.

- $\quad$ internet have able to provide small number of user with ability to move varied from the information easily and thus transformation business, science, education, defense, research, industry, and human interactions.

- Sensor network, in the great rush, be equal significant by providing highest of the physical phenomena around $\mathrm{u}$, leading to their understanding and ultimately the employment of this information for a broad range of applications.

- potential application of sensor network include healthcare monitoring, modern highway, environmental monitoring, and battlefield surveillance or reconnaissance, condition- based maintenance of complex system, and so forth.

\section{UTILIZATION OF WIRELESS SENSOR NETWORK}

The utilization of wireless sensor network is many [2]. They can use in economic and industrial application to monitoring data that would be demanding or expansive to monitor using wired sensor. They can be deployed in a forest area, they would be remain for many year (complete some environmental variable) without the needs to Replace/recharge their power supplies. The perimeter about a property and monitor the advancement intruders (passing the information for node to another node). Common application of wireless sensor network includes tracking, controlling, and monitoring. Some of the unique application is object taking, fire detection, nuclear reactor, and traffic monitoring, etc. the common application of a WSN is scattered in a region it is meant to collect data through sensor nodes.

\section{CONCLUSION}

Wireless sensor networks are one of the most emerging fields in research operation. WSN has a important feature of the auditor environment and physical development like as pressure, humidity, temperature etc. In this paper we are discuss different conditions of wireless sensor network and also discussed different type of WSN and their utilization and classified various groups of routing protocol. WSN are used to assemble data from the environment. They constitute small number of sensor nodes and one or more base station. The nodes are connected to the network via wireless communication channels. All nodes have capability to sense the data, and send it to rest of the nodes to base station. These finite nodes are the battery lifetime. The costs of sensor nodes are same as variable, ranging from the less to hundreds of dollars, depends on the complexity of the specific sensor nodes The WSN topology can vary from the star network to an advanced multi-hop network.

\section{ACKNOWLEDGMENT}

It gives us an immense pleasure to acknowledge our deep sense of gratitude to our guide MR. Amit Grover Saheed Bhagat Singh State Technical Campus.

He has been an inspiration and superb guide which helped us to present this work in its present form. Their technical assistance and constructive criticism helped us to give a rational and systematic approach to this paper.

We shall always be indebted to his leamed and thoughtful guidance and supervision.

We extend our sense of gratitude to all those who directly or indirectly helped us during the preparation of this paper.

\section{REFERENCES}

[1]Ian F. Akyildiz, Weilian Su, Yogesh Sankarasubramaniam, and Erdal Cayirci,"A Survey on Sensor Networks"-Aug 2002, IEEE Communications Magazine.

[2] Carlos F. García-Hernández, Pablo H. IbargüengoytiaGonzález, Joaquín García-and Hernández, "Wireless Sensor Networks and Applications: a Survey", IJCSNS International Journal of Computer Science and Network Security,-March 2007 Volume 7,Issue.3, Page(s).208-218.

[3] Fatma Bouabdallah, Nizar Bouabdallah, and Raouf Boutaba , "On Balancing Energy Consumption in Wireless Sensor Networks",-July 2009 IEEE Transactions On Vehicular Technology, Volume. 58, Issue 6, Page(s). 117-123.

[4] Giuseppe Anastasi, Marco Conti, Mario Di Francesco, Andrea Passarella, "Energy Conservation in Wireless Sensor Networks: a Survey,"-May 2009, Volume 7, Issue 3, Pages .537-568

[5] Mayank Saraogi."A Survey of wireless sensor network, EURASIP Journal on Wireless Communications and Networking "-2005, Page(s) .774-788.

[6] Paulo Rogério Pereira, António Grilo, Francisco Rocha, Mário Serafim Nunes, Augusto Casaca, Claude Chaudet, Peter Almström and Mikael Johansson, "End-To-End Reliability In Wireless Sensor Networks:Survey And Research Challenges" International Conference on Wireless Communications, Networking and Mobile Computing, 2007. WiCom 2007, Page(s).2771 - 2774.

[7] Zheng, Yugui Qu, Baohua Zhao, "Data Aware Clustering for Data Gathering in Wireless Sensor Networks", International Conference on Networks Security, Wireless Communications and Trusted Computing, 2009, Volume.1, Page(s). 192-214.

[8] Dinesh Kumar Gupta "A Review on Wireless Sensor Network", International conference on recant trends in applied sciences with engineering Application, 2013, volume 3.No1.

[8]S.S. Iyengar, D.N. Jayasimha, D. Nadig, A versatile architecture for the distributed sensor integration problem, IEEE Trans. Comput. 43 (2) (1994) 175-185.

[9] D.N. Jayasimha, S.S. Iyengar, R.L. Kashyap, Information integration and synchronization in distributed sensor networks, IEEE Trans. Systems, Man, Cybernet. SMC21(21) (1991) 1032- 1043. 
[10] Knoll, J. Meinkoehn, Data fusion using large multi-agent networks: an analysis of network structure and performance, In: Proceedings of the International Conference on Multisensor Fusion and Integration for Intelligent Systems (MFI), Las Vegas, NV, October 2-5 1994, IEEE, pp. 113-120.

[11] L. Prasad, S.S. Iyengar, R.L. Kashyap, R.N. Madan, Functional characterization of sensor integration in distributed sensor networks, IEEE Trans. Systems, Man, Cybernet. SMC-21 (5) (1991) 1082-1087.

[12] R. Wesson, F. Hayes-Roth, J.W. Burge, C. Stasz, C.A. Sunshine, Network structures for distributed situation assessment, IEEE Trans. Systems, Man, Cybernet. SMC11(1) (1981) 5-23.

[13] M.R. Samantham, D.K. Pradhan, The debruijn multiprocessor network: a versatile parallelprocessing and sorting network for vlsi, IEEE Trans. Comput. 38 (4) (1989) 576-581.

[14] LuisJaveir Garcia Villalba, Ana Lucila Sandoval Orozco, Alicia TrivinoCabera and Claudia JacyBarenco Abbas, routing Protocols in Wireless Sensor Networks, Sensors2009.
[15] C.E. Perkins, Ad HocNetworkin g, Addison-Wesley, Reading MA, December 2000.

[16] Rajashree.V.Biradar,V.C.Patil, Dr. S.R. Sawant, Dr. R.R. Mudholkar,Classification and Comparison of Routing Protocols in Wireless Sensor Networks, Special Issue on Ubiquitous Computng Security Systems.

[17] A.D. Birrell, B.J. Nelson, Implementing remote procedure calls, ACM Trans. Comput. Systems 2 (1)(1984) 39-59.

[18] S. Baker, Corba implementation issues, IEE Colloq. (Digest) (007) (1994) 5/1-5/3.

[19] Watson, Omg (object management group) architecture and corba (common object request broker architecture) specification, IEE Colloq.FDigest, (007) (1994) 4/1.

[20] Todd Sundsted, An introduction to agents, Java World, June 1998

[21] H. Qi, S.S. Iyengar, K. Chakrabarty, Multi-resolution data integration using mobile agents in distributed sensor networks, IEEE Transactions on SMC: C (2000), submitted. 